\title{
Depolarizing-Field Effects in Epitaxial Capacitor Heterostructures
}

\author{
N. Strkaljø, ${ }^{1, *,}$ G. De Luca, ${ }^{1, *}$ M. Campanini, ${ }^{2}$ S. Pal, ${ }^{1}$ J. Schaab,${ }^{1}$ C. Gattinoni, ${ }^{1}$ N. A. Spaldin, ${ }^{1}$ \\ M. D. Rossell, ${ }^{2}$ M. Fiebig, ${ }^{1}$ and M. Trassin ${ }^{1, *}$ \\ ${ }^{1}$ Department of Materials, ETH Zurich, CH-8093 Zürich, Switzerland \\ ${ }^{2}$ Electron Microscopy Center, Empa, Swiss Federal Laboratories for Materials Science and Technology, \\ CH-8600 Dübendorf, Switzerland
}

(Received 30 November 2018; revised manuscript received 8 April 2019; published 4 October 2019)

\begin{abstract}
We identify a transient enhancement of the depolarizing field, leading to an unexpected quench of net polarization, during the growth of a prototypical metal-ferroelectric-metal epitaxial system made of $\mathrm{BaTiO}_{3}$ and $\mathrm{SrRuO}_{3}$. Reduced conductivity and, hence, charge screening efficiency in the early growth stage of the $\mathrm{SrRuO}_{3}$ top electrode promotes a breakdown of ferroelectric $\mathrm{BaTiO}_{3}$ into domains. We demonstrate how a thermal annealing procedure can recover the single-domain state. By tracking the polarization state in situ, using optical second harmonic generation, we bring new understanding to interface-related electrostatic effects in ferroelectric capacitors.
\end{abstract}

DOI: $10.1103 /$ PhysRevLett.123.147601

The demand for ever-smaller and energy-efficient devices drives the development of ultrathin ferroelectrics with a robust polarization state and reliable switching properties [1-5]. However, the macroscopic polarization can be lost when the ferroelectric is implemented into the required metal-ferroelectric-metal thin-film capacitor heterostructure $[6,7]$ since interface-related effects can drastically alter the polarization behavior [8-12]. For example, uncompensated bound surface charges at epitaxial ferroelectric interfaces result in a depolarizing field, which can trigger a drop of the ferroelectric Curie temperature $\left(T_{c}\right)$ or nanoscale domain splitting [13-17]. Furthermore, ferroelectric layers are usually grown below $T_{c}$ [18-21], so that their polarization state is set during the heterostructure growth [22-24]. Here the deposition process and the accompanying transient electrostatic effects at the metal-ferroelectric interface govern the resulting polarization state. Hence, understanding the growth dynamics and promoting the involved depolarization fields towards a robust remnant polarization and a controlled domain distribution in the ultrathin regime poses a key challenge on the way to ultrathin functional ferroelectric heterostructures and devices [25].

In this Letter, we identify microscopic physical mechanisms determining the depolarizing field and domain formation in a prototypical metal-ferroelectric-metal epitaxial heterostructure, $\mathrm{SrRuO}_{3}\left|\mathrm{BaTiO}_{3}\right| \mathrm{SrRuO}_{3}(\mathrm{SRO}|\mathrm{BTO}| \mathrm{SRO})$. We track the evolution of the ferroelectric order during the deposition by laser-optical in situ second harmonic generation (ISHG) [24]. We find that the first unit cells of the top electrode exhibit reduced conductivity and, hence, insufficient charge screening. This triggers a ferroelectric multidomain breakdown manifesting as net-polarization quench. The unprecedented insight into the growth process allows us to develop a procedure controlling the influence of the depolarizing field by thermal annealing. While previous thin-film studies on the depolarizing field in ferroelectrics are restricted to uncapped specimens or periodic domain patterns [14,21], we reveal explicit mechanisms controlling the net polarization of ultrathin ferroelectrics in capacitor heterostructures. This is all the more important since any metallic layer in a heterostructure begins its existence as ultrathin film, becoming "thick" only upon ongoing deposition. Our findings support the development of oxide electronics because they shed light on a hitherto inaccessible yet crucial aspect: the evolution of the oxide multilayer heterostructure, right where its functionalities are coined with the deposition of the first monolayers.

We study the polarization state in a model ferroelectric capacitor system composed of a ferroelectric BTO thin film between two metallic SRO electrodes. Uniaxial ferroelectric BTO (001) films with a thickness of 30 unit cells (u.c.) were grown by pulsed laser deposition (PLD) on (001)oriented $\mathrm{SrTiO}_{3}$ (STO) buffered by 10 u.c. of SRO. The substrate exerts $\approx 2.2 \%$ compressive strain, increasing the ferroelectric Curie temperature $\left(T_{c}\right)$ above the growth temperature $\left(T_{\text {growth }}=650^{\circ} \mathrm{C}\right)$ [19]; further details on growth conditions are available in Ref. [26]. Room-temperature ferroelectric properties were confirmed using piezoresponse force microscopy (PFM). The high-quality singledomain as-grown state of the BTO and its local switching behavior are depicted in Figs. 1(a) and 1(b).

We monitor the ferroelectric response during the thinfilm deposition using ISHG as a noninvasive detection technique. SHG denotes frequency doubling of a light wave in a material. This process is sensitive to the loss of inversion symmetry and therefore occurs with the emergence of ferroelectric order [46-48]. The ISHG signal is measure in $45^{\circ}$ reflection geometry and its polarization is chosen such that it detects the out-of-plane component of 
(a)

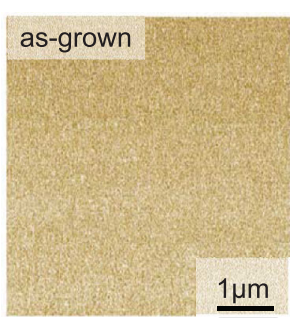

(c)

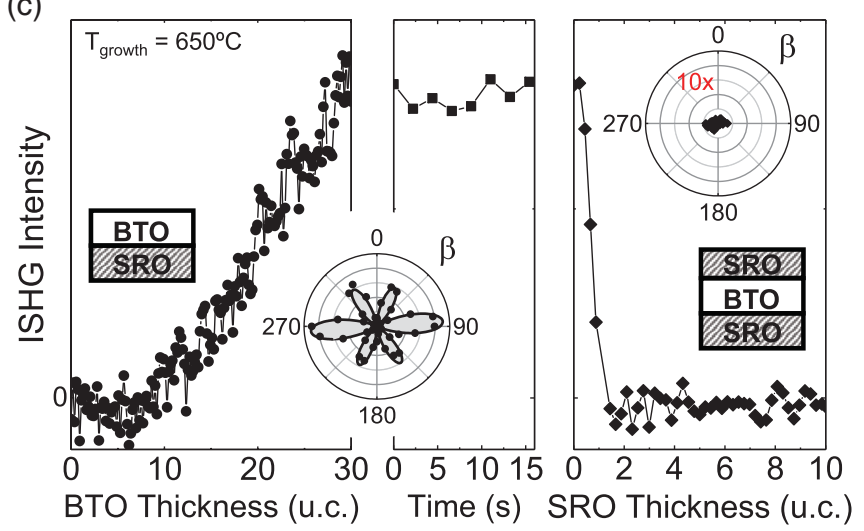

FIG. 1. Evolution of polarization during SRO|BTO|SRO capacitor design. (a)-(b) PFM phase images showing out-of-plane contrast of compressively strained BTO grown on SRO-buffered STO, with $\pm 10 \mathrm{~V}$ applied to the scanning tip. (c) ISHG signal during the growth of BTO on SRO-buffered STO (left panel) at $T_{\text {growth }}=650^{\circ} \mathrm{C}$, during hold time after the completion of the BTO layer (middle panel) and during subsequent deposition of the top SRO layer (right panel). The insets show the ISHG anisotropy obtained by simultaneously varying the polarization angle $(\beta)$ of the incident fundamental $(1200 \mathrm{~nm})$ and the detected SHG $(600 \mathrm{~nm})$ light. The simulations are plotted as continuous lines. Note that in the right panel no signal is recovered after cooling to room temperature.

the ferroelectric polarization emerging in the BTO during the deposition process [49]. Reflection high energy electron diffraction (RHEED) is performed simultaneously with ISHG to ensure the growth quality and to calibrate the ISHG yield to the thickness with u.c. accuracy [26].

Figure 1(c) presents the ISHG signal during the deposition of the BTO and the SRO cap layer of the SRO|BTO|SRO $\mid$ STO heterostructure. The left panel confirms an onset of the ferroelectric BTO polarization at a critical thickness of 5 u.c. on SRO $\|$ STO [24,50]. The ISHG anisotropy measurement in the inset is characteristic of the tetragonal point-group symmetry $4 \mathrm{~mm}$ of the BTO with an out-of-plane orientation of the spontaneous polarization. The ISHG data combined with ex situ PFM-phase measurements [Fig. 1(a)] show the single-domain nature of our BTO films. This indicates that the depolarizing field in BTO is suppressed by sufficient charge screening exerted by the 10 u.c. bottom SRO and the oxygen-rich growth atmosphere [51-54].

We now follow the BTO polarization state during the subsequent deposition of the SRO cap layer. Most strikingly, the ISHG intensity drops to zero with the growth of only 2 u.c. of SRO, see right panel of Fig. 1(c) (with an error of \pm 0.5 u.c. [26,55]). This net-polarization quench is startling as it suggests the emergence of a strong depolarizing field, which is in contradiction to the expected metallic nature of the SRO. The ISHG drop cannot be attributed to probe-laser damage because sustained irradiation of the completed BTO film does not yield an ISHG decrease (middle panel). Linear absorption of the ISHG light passing an SRO cap layer of 2 u.c. results in an intensity decrease of less than 10\% [24] and cannot explain its complete quench either.

The ISHG signal breakdown could result from strain relaxation and/or chemical disorder at the interface, which might lower $T_{c}$ and push the BTO into the paraelectric phase. Therefore, we investigated the strain state, the local atomic structure, and the chemical composition of the SRO $|\mathrm{BTO}| \mathrm{SRO} \|$ STO trilayer. For this, a sphericalaberration-corrected FEI Titan Themis microscope operated at $300 \mathrm{kV}$ and equipped with ChemiSTEM technology was used; see Ref. [26] for more details. The post-deposition scanning-transmission-electron-microscopy (STEM) analysis in Fig. 2 reveals that the BTO layer sustains the in-plane strain state from the substrate. Hence, we rule out a strain-relaxation-induced drop of $T_{c}$ as a cause of the ISHG quench. Alternatively, pinned dipoles at $\mathrm{RuO}_{2} \mid \mathrm{BaO}-$ terminated interfaces were reported to suppress the polarization of BTO [56]. For verifying this, the atomic species at the interfaces were resolved using atomic-resolution energydispersive X-ray (EDX) spectroscopy. The sharp SRO|BTO interface shows very little interdiffusion. In addition, we found that both interface terminations are $\mathrm{SrO} \mid \mathrm{TiO}_{2}$, ultimately excluding the role of interface chemistry in the ISHG loss.

Exclusion of the aforementioned mechanisms leaves electrostatic effects [57,58] as likely explanation of the ISHG quench. Insufficient charge screening by the top SRO electrode could result in an enhanced depolarizing field and concomitant multidomain or paraelectric-state formation and thus, net-polarization breakdown. However, as the bottom electrode shows, 10 u.c. of SRO screen the BTO charges sufficiently, according to the single domain state of the BTO layer in Fig. 1(a).

We therefore need to consider the charge screening efficiency of an SRO top electrode below 10 u.c. thickness, a stage that our heterostructure inevitably undergoes during the deposition of the final 10 u.c. electrode. We used density functional theory (DFT) to explore the SRO metallicity in the ultrathin regime. We calculated the density of states (DOS) at the Fermi energy $\left(E_{F}\right)$ as a function of the SRO thickness. Calculations were performed with the PBEsol functional [59] using the vASP code [60] with the PAW approach [61]. Plane waves were cut off at an energy of $500 \mathrm{eV}$ and a $6 \times 6 \times 6$ Monkhorst-Pack grid was used for a single perovskite unit cell. A Hubbard- $U$ term [62] with $U_{\text {eff }}=2.0 \mathrm{eV}$ was applied to the $d$ orbitals of Ru. Our top 
(a)

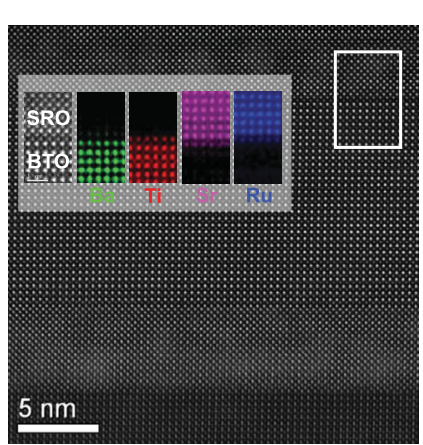

(b)

(c)

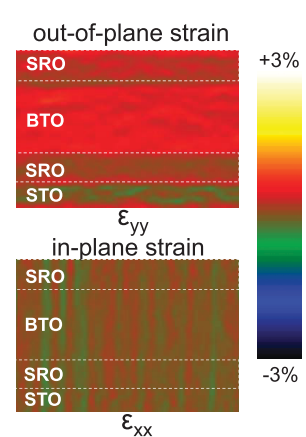

(d)

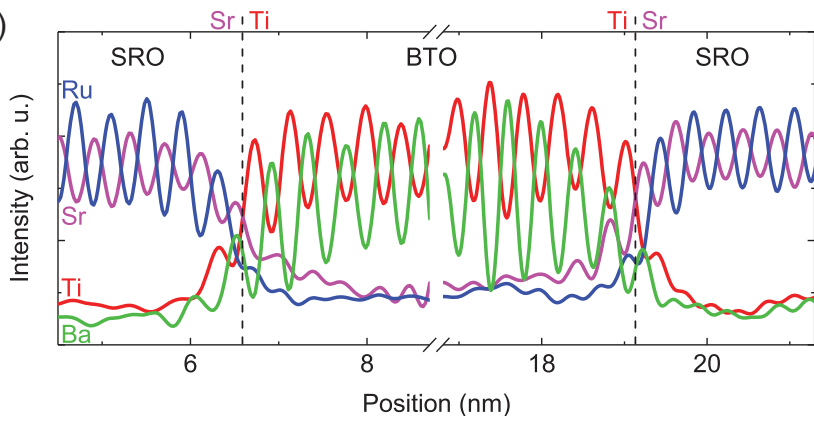

FIG. 2. Analysis of strain state and atomic species at the interfaces of SRO $|\mathrm{BTO}| \mathrm{SRO}$ capacitor. (a) Atomically resolved structure using high-angle annular dark-field STEM. The inset shows the EDX analysis indicating an atomically sharp BTOISRO interface with very limited interdiffusion. (b)-(c) Strain maps showing the out-of-plane (b) and in-plane (c) lattice parameter variation relative to the STO substrate lattice constant. The SRO|BTO|SRO capacitor is coherently strained in-plane to the SRO substrate excluding strain relaxation as the polarization suppression mechanism. (d) Interface termination by EDX spectroscopy. Both SRO $|\mathrm{BTO}| \mathrm{SRO}$ interfaces have $\mathrm{SrO} \mid \mathrm{TiO}_{2}$ termination which is not detrimental to the polarization state [56]. Hence, the preservation of strain, minimal degree of interdiffusion, and interface chemistry suggest electrostatic effects as an explanation for the net-polarization quench in Fig. 1.

SRO|BTO electrode is coherently strained to the STO substrate; see Figs. 2(b) and 2(c). Furthermore, the interface termination at the top $\mathrm{SRO} \mid \mathrm{BTO}$ is $\mathrm{SrO} \mid \mathrm{TiO}_{2}$; see Fig. 2(d). In the calculations, we therefore considered an SRO|BTO heterostructure with the experimentally relevant in-plane STO substrate lattice parameter, $\mathrm{SrO} \mid \mathrm{TiO}_{2}$ interface termination, and upwards ferroelectric polarization of the BTO layer. A heterostructure of 5 u.c. BTO and $n$ u.c. SRO was relaxed. The SRO is in the $P 4 / \mathrm{mbm}$ space group while the BTO has neither octahedral rotations nor tilts [63]. Forces were converged to $5 \times 10^{-3} \mathrm{eV} / \AA^{2}$. We found that an insulating antiferromagnetic configuration is preferred below a thickness of 2 u.c., while the metallic ferromagnetic configuration is preferred at 2 u.c. and higher thicknesses; see Fig. 3(a). In line with this, previous reports on SRO showed a thickness-related metal-insulator transition in similar systems [64-66]. Experimentally, we extracted the optical conductivity of the 1,2 , and 10 u.c. SRO cap layers in the SRO|BTO|SRO $\mid$ STO system from reflectance spectra (a)

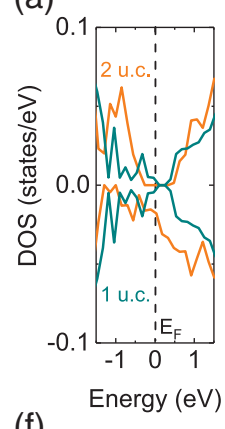

(b)

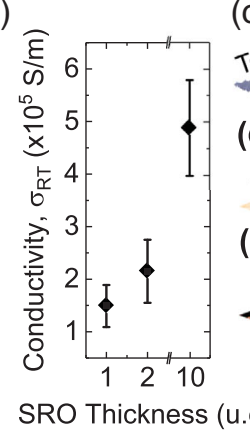

(c)

(f)
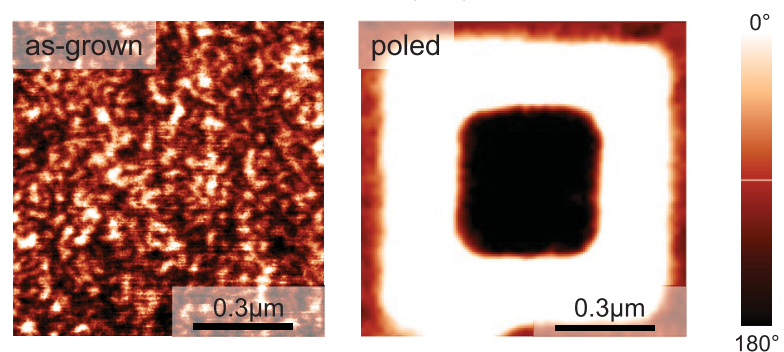

FIG. 3. Origin of the BTO net-polarization quench. (a) DFT calculations compare the DOS at $E_{F}$ for different thicknesses of SRO. The antiferromagnetic 1 u.c. thick SRO film is insulating, as shown by the zero DOS at $E_{F}$. The ferromagnetic 2 u.c. thick SRO and thicker films are metallic according to the finite DOS at $E_{F}$. (b) Terahertz time-domain spectroscopy measurements show a continuous decrease in top SRO conductivity $\sigma_{\mathrm{RT}}$ with layer thickness. (c)-(e) Topography and corresponding out-of-plane PFM and conductive atomic force microscopy (cAFM) data after nanomachining away the SRO top electrode to expose the BTO. The removal is visible as a step in the topography image (c), as an enhancement of PFM signal (d), and as suppressed conduction (e) in the exposed BTO area. (f) Resonant PFM of the as-grown exposed BTO surface reveals a multidomain pattern in the PFM phase. Voltage tip poling in the area on the right recovers a singledomain state, confirming the preservation of ferroelectricity in the BTO. The bright area is down-polarized with a positive tip voltage and the dark area is up-polarized with a negative tip voltage.

measured by terahertz time-domain spectroscopy [26-29]. Our results in Fig. 3(b) are in agreement with the previous measurements on SRO films [30,31] and show a pronounced reduction of conductivity towards thinner SRO layers. The reduction in conductivity together with the diminishing thickness leads to a continuous decrease in screening efficiency from bulklike to a monolayer. The poorly conducting 1-2 u.c. thick SRO abruptly isolates the BTO from the charge-screening oxygen-rich growth atmosphere $[53,54]$ and enhances the depolarizing field.

For a direct verification of the depolarizing-field-induced multidomain breakdown, we image the domain state of our heterostructures by PFM. Using the ISHG real-time feedback, we deposited the amount of SRO required for a netpolarization quench, subsequently removing the SRO by nanomachining with a diamond-coated PFM tip. There is a risk that the mechanical forces in this procedure might 
affect the domain state. However, in earlier tomographic PFM characterization, this was not observed [32]. The absence of BTO surface degradation or mechanical switching induced by this process [67] was verified by AFM in the nanomachined area [26]. By exposing the BTO, we can probe its domain state by high-resolution resonant PFM and confirm that the cap SRO deposition has led to the formation of a $180^{\circ}$ domain maze pattern with an average domain width of $30 \pm 10 \mathrm{~nm}$ [68]. Preservation of the ferroelectric nature of this state is evidenced by reversible tip poling of the polarization with $\pm 10 \mathrm{~V}$ in the exposed area; see PFM phase images in Fig. 3(f).

How can we bypass the transient depolarizing field enhancement and thus recover the single-domain ferroelectric state of the BTO in the final capacitor heterostructure? Wide-area electric-field poling via the SRO electrodes shows limited efficiency because of pinned and randomly oriented domains at local defects or interfaces [69-71]. As an alternative, we should be able to delete the ferroelectric state entirely by a heating cycle through $T_{c}$ and recover the single-domain ferroelectric state of the BTO on cooling, provided that the thermal treatment is performed once the SRO cap layer reaches the thickness at which it is sufficiently metallic. When cooling the sample from above $T_{c}$, the buildup of the transient depolarizing field is now avoided, and a single-domain state should result.

For verifying this approach, we use strain engineering to reduce $T_{c}$ to reach an experimentally accessible value and to avoid sample deterioration by the annealing cycle. We consider three different substrates: STO (lattice mismatch $\eta=-2.2 \%$ ), (110)-oriented $\mathrm{DyScO}_{3}$ (DSO) $(\eta=-1.2 \%)$ and (110)-oriented $\mathrm{GdScO}_{3}(\mathrm{GSO})(\eta=$ $-0.6 \%)$. The ISHG signal during the BTO growth in Fig. 4(a) confirms the single-domain ferroelectric state of the BTO for all three substrates with lower polarization for lower epitaxial compressive stress. In Fig. 4(b), temperature-dependent SHG shows the reduction of $T_{c}$ with the reduction of compressive stress, finally leading to an accessible $T_{c}=720^{\circ} \mathrm{C}$ on GSO.

We therefore apply the annealing cycle to the SRO|BTO|SRO $\|$ GSO. The ISHG anisotropy in Fig. 4(c) reveals that the single-domain state is quenched, as with the STO substrate, by the deposition of the first layers of SRO cap electrode. After completing the heating cycle through $T_{c}$, the ISHG yield and its light polarization dependence are completely restored [26]. The annealing process is schematized in Fig. 4(d). The result of the annealing experiment is another strong confirmation that the transient enhancement of the depolarizing field, caused by imperfect charge screening in the early stage of the top electrode deposition, explains the multidomain breakdown, rather than chemical intermixing or termination effects, that would not be eliminated by the annealing. More importantly, we see that annealing is an attractive alternative to
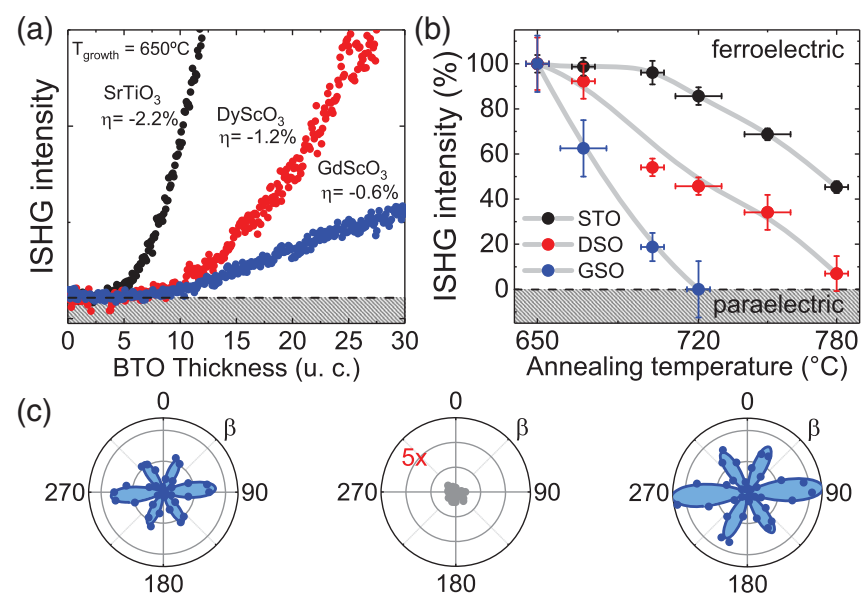

(d)

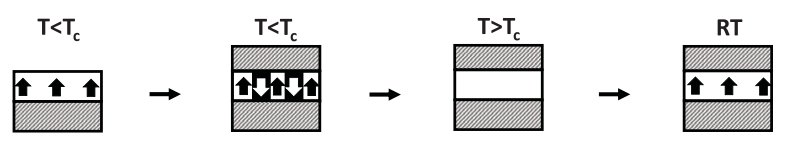

FIG. 4. Domain engineering in $\mathrm{SRO}|\mathrm{BTO}| \mathrm{SRO}$ by thermal annealing. (a) ISHG signal during BTO growth on SRO buffered (001)-STO $(\eta=-2.2 \%)$, (110)-DSO $(\eta=-1.2 \%)$, and (110)GSO ( $\eta=-0.6 \%)$ substrates. (b) ISHG intensity as a function of temperature for different substrates, relative to the value at the end of growth $\left(T_{\text {growth }}=650{ }^{\circ} \mathrm{C}\right)$. The decrease of $T_{c}$ with decreasing lattice mismatch is used to suppress $T_{c}$ for SRO|BTO|SRO on GSO sufficiently to heat it above $T_{c}$ and reset the polarization state of the completed heterostructure. (c) ISHG anisotropy measurements at different stages of the annealing process. Single-domain polarization after the BTO $\mid \mathrm{SRO} \| \mathrm{GSO}$ growth (left). After the SRO capping, the signal yield is zero because of destructive multidomain SHG interference (middle). Thermal annealing recovers the initial ISHG signal and, hence, a singledomain state (right). Absolute ISHG intensities from BTO change with temperature and with the thickness of the SRO capping layer which absorbs the fundamental light. (d) Sketch of the annealing process. Data points in (b)-(c) were taken after realignment for temperature-induced mechanical drifts.

the notoriously difficult electric-field poling in establishing an as-grown single-domain ferroelectric heterostructure in the ultrathin regime.

In summary, we have tracked the polarization state of a prototypical ferroelectric, $\mathrm{BaTiO}_{3}$, throughout deposition of a metallic $\mathrm{SrRuO}_{3}$ top electrode. Laser-optical in situ second harmonic generation, supported by density functional theory and terahertz time-domain spectroscopy, reveals a transient enhancement of the depolarization field because of the "bad" metallic nature of ultrathin $\mathrm{SrRuO}_{3}$. We thus pinpoint insufficient charge screening as the origin of the resulting multidomain breakdown of the ferroelectric net polarization.

Because most of the commonly used metal-oxide electrodes exhibit a thickness-dependent conductivity [64-66,72-74], our conclusions are independent of the choice of the oxide material and the epitaxial growth technique. The ability to tune the depolarizing field during the deposition goes beyond the capacity to engineer the 
domain formation during growth. For instance, interfacial charge screening is a key ingredient in enhanced ferroelectric behavior [17] and the recently reported negativecapacitance heterostructures [75]. Therefore, our work, bringing awareness of the electrostatics dynamics during the heterostructure design, will stimulate not only the investigation of complex electrostatics in ultrathin ferroelectric heterostructures and superlattices but also at polar surfaces and interfaces in general.

The authors thank E. Gradauskaite for experimental assistance with scanning probe microscopy. This research was supported by the EU European Research Council (Advanced Grant No. 694955-INSEETO). M. F. thanks ETH Zurich and Center for Emergent Matter Science (CEMS) at RIKEN for support of his research sabbatical. M. C. and M. D. R. acknowledge support by the Swiss National Science Foundation under Project No. 200021_175926. S. P. acknowledges support by ETH Career Seed Grant No. SEED-17 18-1. C. G. acknowledges support by the European Union's Horizon 2020 research and innovation program under the Marie Skłodowska-Curie Grant Agreement No. 744027. The DFT calculations were supported by a grant from the Swiss National Supercomputing Centre (CSCS) under Project ID s798.

All authors discussed the results and contributed to the completion of the manuscript. N. S. and G. D. L. performed the thin film growth, SHG measurements, and developed the SHG fitting model. M. C. and M. D. R. carried out the STEM analysis. J. S., M. T., and N. S. performed the PFM investigation. S.P. performed the terahertz time-domain spectroscopy. C. G. and N. A. S. provided the DFT calculations. M. T. designed the experiment and supervised the work with M. F.

*These authors contributed equally to this work.

nives.strkalj@mat.ethz.ch

*morgan.trassin@mat.ethz.ch

[1] L. W. Martin and A. M. Rappe, Nat. Rev. Mater. 2, 16087 (2016).

[2] K. M. Rabe, M. Dawber, C. Lichtensteiger, C. H. Ahn, and J.-M. Triscone, in Physics of Ferroelectrics: A Modern Perspective (Springer, Berlin, 2007), pp. 1-30.

[3] A. Chanthbouala, A. Crassous, V. Garcia, K. Bouzehouane, S. Fusil, X. Moya, J. Allibe, B. Dlubak, J. Grollier, S. Xavier, C. Deranlot, A. Moshar, R. Proksch, N. D. Mathur, M. Bibes, and A. Barthélémy, Nat. Nanotechnol. 7, 101 (2012).

[4] A. Q. Jiang, X. J. Meng, D. W. Zhang, M. H. Park, S. Yoo, Y. J. Kim, J. F. Scott, and C. S. Hwang, Sci. Rep. 5, 14618 (2015).

[5] M. Dawber, K. M. Rabe, and J. F. Scott, Rev. Mod. Phys. 77, 1083 (2005).

[6] J. Junquera and P. Ghosez, Nature (London) 422, 506 (2003).

[7] D. J. Kim, J. Y. Jo, Y. S. Kim, Y. J. Chang, J. S. Lee, J.-G. Yoon, T. K. Song, and T. W. Noh, Phys. Rev. Lett. 95, 237602 (2005).
[8] A. K. Tagantsev and G. Gerra, J. Appl. Phys. 100, 051607 (2006).

[9] C.-L. Jia, V. Nagarajan, J.-Q. He, L. Houben, T. Zhao, R. Ramesh, K. Urban, and R. Waser, Nat. Mater. 6, 64 (2007).

[10] A. Cano and A. P. Levanyuk, arXiv:1408.2373.

[11] Y. J. Shin, Y. Kim, S.-J. Kang, H.-H. Nahm, P. Murugavel, J. R. Kim, M. R. Cho, L. Wang, S. M. Yang, J.-G. Yoon, J.-S. Chung, M. Kim, H. Zhou, S. H. Chang, and T. W. Noh, Adv. Mater. 29, 1602795 (2017).

[12] S. K. Streiffer, J. A. Eastman, D. D. Fong, C. Thompson, A. Munkholm, M. V. Ramana Murty, O. Auciello, G. R. Bai, and G. B. Stephenson, Phys. Rev. Lett. 89, 067601 (2002).

[13] A. M. Bratkovsky and A. P. Levanyuk, Phys. Rev. Lett. 84, 3177 (2000).

[14] C. Lichtensteiger, S. Fernandez-Pena, C. Weymann, P. Zubko, and J.-M. Triscone, Nano Lett. 14, 4205 (2014).

[15] C. Lichtensteiger, M. Dawber, N. Stucki, J.-M. Triscone, J. Hoffman, J.-B. Yau, C. H. Ahn, L. Despont, and P. Aebi, Appl. Phys. Lett. 90, 052907 (2007).

[16] C.-G. Duan, R. F. Sabirianov, W.-N. Mei, S. S. Jaswal, and E. Y. Tsymbal, Nano Lett. 6, 483 (2006).

[17] G. Liu, J. Chen, C. Lichtensteiger, J.-M. Triscone, P. Aguado-Puente, J. Junquera, and N. Valanoor, Adv. Electron. Mater. 2, 1500288 (2016).

[18] D. G. Schlom, L.-Q. Chen, C.-B. Eom, K. M. Rabe, S. K. Streiffer, and J.-M. Triscone, Annu. Rev. Mater. Res. 37, 589 (2007).

[19] K. J. Choi, M. Biegalski, Y. L. Li, A. Sharan, J. Schubert, R. Uecker, P. Reiche, Y. B. Chen, X. Q. Pan, V. Gopalan, L.-Q. Chen, D. G. Schlom, and C. B. Eom, Science 306, 1005 (2004).

[20] A. R. Damodaran, E. Breckenfeld, Z. Chen, S. Lee, and L. W. Martin, Adv. Mater. 26, 6341 (2014).

[21] D. D. Fong, G. B. Stephenson, S. K. Streiffer, J. A. Eastman, O. Auciello, P. H. Fuoss, and C. Thompson, Science 304, 1650 (2004).

[22] J. Sinsheimer, S. J. Callori, B. Ziegler, B. Bein, P. V. Chinta, A. Ashrafi, R. L. Headrick, and M. Dawber, Appl. Phys. Lett. 103, 242904 (2013).

[23] B. Bein, H.-C. Hsing, S. J. Callori, J. Sinsheimer, P. V. Chinta, R. L. Headrick, and M. Dawber, Nat. Commun. 6, 10136 (2015).

[24] G. De Luca, N. Strkalj, S. Manz, C. Bouillet, M. Fiebig, and M. Trassin, Nat. Commun. 8, 1419 (2017).

[25] N. A. Spaldin and R. Ramesh, Nat. Mater. 18, 203 (2019).

[26] See Supplemental Material at http://link.aps.org/ supplemental/10.1103/PhysRevLett.123.147601 for growth conditions, RHEED patterns, and time-dependent intensity during the growth of SROIBTOISRO confirming the twodimensional growth in Figs. S1 and S2; AFM topography scans of 1, 2, and 10 u.c. thick top SRO layers in Fig. S3; STEM polarization analysis in Fig. S4; details of the terahertz time-domain spectroscopy method and spectroscopy results (Figs. S5-S7); details on the absence of correlation between topography and high-resolution resonant PFM in the nanomachined area (Fig. S8); and time-dependent ISHG intensity at different steps of the annealing process in Fig. S9. All this includes Refs. [27-32] and [33-45]. 
[27] D. M. A. Mackenzie, P. R. Whelan, P. Bøggild, P. U. Jepsen, A. Redo-Sanchez, D. Etayo, N. Fabricius, and D. H. Petersen, Opt. Express 26, 9220 (2018).

[28] S. Nashima, O. Morikawa, K. Takata, and M. Hangyo, Appl. Phys. Lett. 79, 3923 (2001).

[29] G. Grüner, Millimeter and Submillimeter Wave Spectroscopy of Solids (Springer, Berlin Heidelberg, 1998).

[30] D. Geiger, U. S. Pracht, M. Dressel, J. Mravlje, M. Schneider, P. Gegenwart, and M. Scheffler, Phys. Rev. B 93, 165131 (2016).

[31] P. Kostic, Y. Okada, N. C. Kollins, Z. Schlesinger, J. W. Reiner, L. Klein, A. Kapitulnik, T. H. Geballe, and M. R. Beasley, Phys. Rev. Lett. 81, 2498 (1998).

[32] J. J. Steffes, R. A. Ristau, R. Ramesh, and B. Huey, Proc. Natl. Acad. Sci. U.S.A. 116, 2413 (2019).

[33] S. Kobayashi, K. Inoue, T. Kato, Y. Ikuhara, and T. Yamamoto, J. Appl. Phys. 123, 064102 (2018).

[34] V. Lucarini, J. J. Saarinen, K.-E. Peoponen, and E. M. Vartiainen, Kramers-Kronig Relations in Optical Materials Research (Springer, Berlin Heidelberg, 2005).

[35] C.-W. Chen, Y.-C. Lin, C.-H. Chang, P. Yu, J.-M. Shieh, and C.-L. Pan, IEEE J. Quantum Electron. 46, 1746 (2010).

[36] N. Matsumoto, T. Hosokura, T. Nagashima, and M. Hangyo, Opt. Lett. 36, 265 (2011).

[37] F. D. J. Brunner, A. Schneider, and P. Günther, Opt. Express 17, 20684 (2009).

[38] M. Bass, Handbook of Optics (McGraw-Hill, New York, 1995), Vol. I.

[39] B. G. Alberding, R. Thurber, and E. J. Heilweil, J. Opt. Soc. Am. B 34, 1392 (2017).

[40] P. Kužel and F. Kadlec, C.R. Phys. 9, 197 (2008).

[41] P. Kužel, F. Kadlec, H. Němec, R. Ott, E. Hollmann, and N. Klein, Appl. Phys. Lett. 88, 102901 (2006).

[42] J. Han, F. Wan, Z. Zhu, and W. Zhang, Appl. Phys. Lett. 90, 031104 (2007).

[43] G. Koster, L. Klein, W. Siemons, G. Rijnders, J. S. Dodge, C.-B. Eom, D. H. A. Blank, and M. R. Beasley, Rev. Mod. Phys. 84, 253 (2012).

[44] R. Kinjo, X. Weiming, I. Kawayama, H. Murakami, and M. Tonouchi, in Proceedings of the 2011 International Conference in Infrared, Millimeter, and Terahertz Waves, Houston (2011), https://doi.org/10.1109/irmmw-THz.2011 .6104855 .

[45] V. Garcia, M. Bibes, L. Bocher, S. Valencia, F. Kronast, A. Crassous, X. Moya, S. Enouz-Vedrenne, A. Gloter, D. Imhoff, C. Deranlot, N. D. Mathur, S. Fusil, K. Bouzehouane, and A. Barthélémy, Science 327, 1106 (2010).

[46] M. Fiebig, V. V. Pavlov, and R. V. Pisarev, J. Opt. Soc. Am. B 22, 96 (2005).

[47] S. A. Denev, T. T. A. Lummen, E. Barnes, A. Kumar, and V. Gopalan, J. Am. Ceram. Soc. 94, 2699 (2011).

[48] J.-Y. Chauleau, E. Haltz, C. Carrétéro, S. Fusil, and M. Viret, Nat. Mater. 16, 803 (2017).

[49] J. Nordlander, G. De Luca, N. Strkalj, M. Fiebig, and M. Trassin, Appl. Sci. 8, 570 (2018).

[50] G. Gerra, A. K. Tagantsev, N. Setter, and K. Parlinski, Phys. Rev. Lett. 96, 107603 (2006).

[51] R. V. Wang, D. D. Fong, F. Jiang, M. J. Highland, P. H. Fuoss, C. Thompson, A. M. Kolpak, J. A. Eastman,
S. K. Streiffer, A. M. Rappe, and G. B. Stephenson, Phys. Rev. Lett. 102, 047601 (2009).

[52] D. D. Fong, A. M. Kolpak, J. A. Eastman, S. K. Streiffer, P. H. Fuoss, G. B. Stephenson, C. Thompson, D. M. Kim, K. J. Choi, C. B. Eom, I. Grinberg, and A. M. Rappe, Phys. Rev. Lett. 96, 127601 (2006).

[53] M. J. Highland, T. T. Fister, D. D. Fong, P. H. Fuoss, C. Thompson, J. A. Eastman, S. K. Streiffer, and G. B. Stephenson, Phys. Rev. Lett. 107, 187602 (2011).

[54] G. B. Stephenson and M. J. Highland, Phys. Rev. B 84, 064107 (2011).

[55] G. Rijnders and D. H. A. Blank, Appl. Phys. Lett. 84, 505 (2004).

[56] H. Lu, X. Liu, J. D. Burton, C.-W. Bark, Y. Wang, Y. Zhang, D. J. Kim, A. Stamm, P. Lukashev, D. A. Felker, C. M. Folkman, P. Gao, M. S. Rzchowski, X. Q. Pan, C. B. Eom, E. Y. Tsymbal, and A. Gruverman, Adv. Mater. 24, 1209 (2012).

[57] C. T. Black and J. J. Welser, IEEE Trans. Electron Devices 46, 776 (1999).

[58] M. F. Chisholm, W. Luo, M. P. Oxley, S. T. Pantelides, and H. N. Lee, Phys. Rev. Lett. 105, 197602 (2010).

[59] J. P. Perdew, A. Ruzsinszky, G. I. Csonka, O. A. Vydrov, G. E. Scuseria, L. A. Constantin, X. Zhou, and K. Burke, Phys. Rev. Lett. 100, 136406 (2008).

[60] G. Kresse and J. Hafner, Phys. Rev. B 47, 558 (1993).

[61] G. Kresse and D. Joubert, Phys. Rev. B 59, 1758 (1999).

[62] S. L. Dudarev, G. A. Botton, S. Y. Savrasov, C. J. Humphreys, and A. P. Sutton, Phys. Rev. B 57, 1505 (1998).

[63] M. Verissimo-Alves, P. García-Fernández, D. I. Bilc, P. Ghosez, and J. Junquera, Phys. Rev. Lett. 108, 107003 (2012).

[64] D. Toyota, I. Ohkubo, H. Kumigashira, M. Oshima, T. Ohnishi, M. Lippmaa, M. Takizawa, A. Fujimori, K. Ono, M. Kawasaki, and H. Koinuma, Appl. Phys. Lett. 87, 162508 (2005).

[65] J. Xia, W. Siemons, G. Koster, M. R. Beasley, and A. Kapitulnik, Phys. Rev. B 79, 140407 (2009).

[66] X. Shen, X. Qiu, D. Su, S. Zhou, A. Li, and D. Wu, J. Appl. Phys. 117, 015307 (2015).

[67] H. Lu, C.-W. Bark, D. Esque de los Ojos, J. Alcala, C. B. Eom, G. Catalan, and A. Gruverman, Science 336, 59 (2012).

[68] A. Schilling, T. B. Adams, R. M. Bowman, J. M. Gregg, G. Catalan, and J. F. Scott, Phys. Rev. B 74, 024115 (2006).

[69] A. M. Bratkovsky and A. P. Levanyuk, Phys. Rev. B 63, 132103 (2001).

[70] P. Zubko, H. Lu, C.-W. Bark, X. Martí, J. Santiso, C.-B. Eom, G. Catalan, and A. Gruverman, J. Phys. Condens. Matter 29, 284001 (2017).

[71] X.-K. Wei, Y. Yang, L. J. McGilly, L. Feigl, R. E. DuninBorkowski, C.-L. Jia, L. Bellaiche, and N. Setter, Phys. Rev. B 98, 020102(R) (2018).

[72] G. Shibata, K. Yoshimatsu, E. Sakai, V. R. Singh, V. K. Verma, K. Ishigami, T. Harano, T. Kadono, Y. Takeda, T. Okane, Y. Saitoh, H. Yamagami, A. Sawa, H. Kumigashira, M. Oshima, T. Koide, and A. Fujimori, Phys. Rev. B 89, 235123 (2014)

[73] Z. Liao, F. Li, P. Gao, L. Li, J. Guo, X. Pan, R. Jin, E. W. Plummer, and J. Zhang, Phys. Rev. B 92, 125123 (2015). 
[74] M. Golalikhani, Q. Lei, R. U. Chandrasena, L. Kasaei, H. Park, J. Bai, P. Orgiani, J. Ciston, G. E. Sterbinsky, D. A. Arena, P. Shafer, E. Arenholz, B. A. Davidson, A. J. Millis, A. X. Gray, and X. X. Xi, Nat. Commun. 9, 2206 (2018).
[75] A. K. Yadav, K. X. Nguyen, Z. Hong, P. G. Fernández, P. Aguado-Puente, C. T. Nelson, S. Das, B. Prasad, D. Kwon, S. Cheema, A. I. Khan, C. Hu, J. Íñiguez, J. Junquera, L-Q. Chen, D. A. Muller, R. Ramesh, and S. Salahuddin, Nature (London) 565, 468 (2019). 